\title{
388.
}

\section{NOTE ON THE COMPOSITION OF INFINITESIMAL ROTATIONS.}

[From the Quarterly Journal of Pure and Applied Mathematics, vol. viII. (1867), pp. $7-10$.

THE following is a solution of a question proposed by me in the last Smith's Prize Examination:

"Show that infinitesimal rotations impressed upon a solid body may be compounded together according to the rules for the composition of forces."

Definition. The "six coordinates" of a line passing through the point $\left(x_{0}, y_{0}, z_{0}\right)$, and inclined at angles $(\alpha, \beta, \gamma)$, to the axes, are

$$
\begin{array}{ll}
a=\cos \alpha, & f=y_{0} \cos \gamma-z_{0} \cos \beta, \\
b=\cos \beta, & g=z_{0} \cos \alpha-x_{0} \cos \gamma, \\
c=\cos \gamma, & h=x_{0} \cos \beta-y_{0} \cos \alpha .
\end{array}
$$

I use, throughout, the term rotation to denote an infinitesimal rotation; this being so,

Lemma 1. A rotation $\omega$ round the line $(a, b, c, f, g, h)$, produces in the point $(x, y, z)$, rigidly connected with the line, the displacements

$$
\begin{aligned}
& \delta x=\omega(\quad . \quad c y-b z+f), \\
& \delta y=\omega(-c x \quad++a z+g), \\
& \delta z=\omega(b x-a y \quad++h) .
\end{aligned}
$$

Lemma 2. Considering in a solid body the point $(x, y, z)$, situate in the line $(a, b, c, f, g, h)$, then for any infinitesimal motion of the solid body, the displacement of the point in the direction of the line is

$$
=a l+b m+c n+f p+g q+h r,
$$


where $l, m, n, p, q, r$ are constants depending on the infinitesimal motion of the solid body.

Hence, first, for a system of rotations

$$
\begin{aligned}
& \omega_{1} \text { about the line }\left(a_{1}, b_{1}, c_{1}, f_{1}, g_{1}, h_{1}\right), \\
& \omega_{2} \quad " \quad\left(a_{2}, b_{2}, c_{2}, f_{2}, g_{2}, h_{2}\right), \\
& \text { \&c. }
\end{aligned}
$$

the displacements of the point $(x, y, z)$, are

$$
\begin{aligned}
& \delta x=\quad y \Sigma c \omega-z \Sigma b \omega+\Sigma f \omega, \\
& \delta y=-x \Sigma c \omega \cdot+z \Sigma a \omega+\Sigma g \omega, \\
& \delta z=x \Sigma b \omega+y \Sigma a \omega . \quad+\Sigma h \omega ;
\end{aligned}
$$

and when the rotations are in equilibrium, the displacements $(\delta x, \delta y, \delta z)$ of any point $(x, y, z)$ whatever must each of them vanish; that is, we must have

$$
\Sigma \omega a=0, \quad \Sigma \omega b=0, \quad \Sigma \omega c=0, \quad \Sigma \omega f=0, \quad \Sigma \omega g=0, \quad \Sigma \omega h=0,
$$

which are therefore the conditions for the equilibrium of the rotations $\omega_{1}, \omega_{2}$, \&c.

Secondly, for a system of forces

$$
\begin{aligned}
& P_{1} \text { along the line }\left(a_{1}, b_{1}, c_{1}, f_{1}, g_{1}, h_{1}\right), \\
& P_{2} \quad " \quad \Rightarrow \quad\left(a_{2}, b_{2}, c_{2}, f_{2}, g_{2}, h_{2}\right), \\
& \text { \&c. }
\end{aligned}
$$

the condition of equilibrium as given by the principle of virtual velocities is

$$
\Sigma P(a l+b m+c n+f p+g q+h r)=0 ;
$$

or, what is the same thing, we must have

$$
\quad \sum P a=0, \quad \Sigma P b=0, \quad \Sigma P c=0, \quad \Sigma P f=0, \quad \Sigma P g=0, \quad \Sigma P h=0,
$$

which are therefore the conditions for the equilibrium of the forces $P_{1}, P_{2}$, \&c.

Comparing the two results we see that the conditions for the equilibrium of the rotations $\omega_{1}, \omega_{2}$, \&c. are the same as those for the equilibrium of the forces $P_{1}, P_{2}$, \&c.; and since, for rotations and forces respectively, we pass at once from the theory of equilibrium to that of composition; the rules of composition are the same in each case.

\section{Demonstration of Lemma 1 .}

Assuming for a moment that the axis of rotation passes through the origin, then for the point $P$, coordinates $(x, y, z)$, the square of the perpendicular distance from the axis is

$$
\begin{array}{r}
=(\quad-y \cos \gamma+z \cos \beta)^{2} \\
+(x \cos \gamma \cdot-z \cos \alpha)^{2} \\
+(-x \cos \beta+y \cos \alpha \cdot)^{2}
\end{array}
$$

C. VI. 
and the expressions which enter into this formula denote as follows; viz. if through the point $P$, at right angles to the plane through $P$ and the axis of rotation, we draw a line $P Q,=$ perpendicular distance of $P$ from the axis of rotation, then the coordinates of $Q$ referred to $P$ as origin are

$$
\begin{array}{r}
-y \cos \gamma+z \cos \beta \\
x \cos \gamma \cdot z \cos \alpha \\
-x \cos \beta+y \cos \alpha .
\end{array}
$$

respectively. Hence the foregoing quantities each multiplied by $\omega$ are the displacements of the point $P$ in the directions of the axes, produced by the rotation $\omega$. Suppose that the axis of rotation (instead of passing through the origin) passes through the point $\left(x_{0}, y_{0}, z_{0}\right)$; the only difference is that we must in the formulæ write $\left(x-x_{0}, y-y_{0}, z-z_{0}\right)$ in place of $(x, y, z)$ : and attending to the significations of the six coordinates $(a, b, c, f, g, h)$ it thus appears that the displacements produced by the rotation are equal to $\omega$ into the expressions

respectively.

$$
\begin{array}{r}
-c y+b z+f \\
c x \cdot-a z+g, \\
-b x+a y \cdot+h
\end{array}
$$

Demonstration of Lemma 2.

For any infinitesimal motion whatever of a solid body, the displacements of the point $(x, y, z)$ in the directions of the axes are

$$
\begin{aligned}
& \delta x=l \quad-r y+q z, \\
& \delta y=m+r x \quad-p z, \\
& \delta z=n-q x+p y .
\end{aligned}
$$

and hence the displacement in the direction of the line $(\alpha, \beta, \gamma)$, is

$$
\delta x \cos \alpha+\delta y \cos \beta+\delta z \cos \gamma,
$$

which, attending to the signification of the six coordinates $(a, b, c, f, g, h)$, is

$$
=a l+b m+c n+f p+g q+h r,
$$

which is the required expression.

It is proper to remark that the last-mentioned expressions of $(\delta x, \delta y, \delta z)$ are in fact the displacements produced by a translation and a rotation. If we assume that every infinitesimal motion of a solid body can be resolved into a translation and a rotation, then, since a translation can be produced by two rotations, every infinitesimal motion of a solid body can be resolved into rotations alone, and the foregoing expressions for the displacements produced by a rotation, combining any number of them and writing $(\Sigma \omega a, \Sigma \omega b, \Sigma \omega c, \Sigma \omega f, \Sigma \omega g, \Sigma \omega h)=(-p,-q,-r, l, m, n)$ respectively, lead to the expressions for the displacements $\delta x, \delta y, \delta z$ produced by the infinitesimal motion of the solid body. 